\title{
Effects of vicarious pain on self-pain perception: investigating the role of awareness
}

\author{
This article was published in the following Dove Press journal: \\ Journal of Pain Research \\ 31 July 2017 \\ Number of times this article has been viewed
}

\author{
Esslin L Terrighena ${ }^{1,2}$ \\ Ge Lu' \\ Wai Ping Yuen' \\ Tatia M C Lee Le $^{1-4}$ \\ Kati Keuper ${ }^{1,2,5}$ \\ 'Department of Psychology, \\ Laboratory of Neuropsychology, \\ The University of Hong Kong, \\ Hong Kong; ${ }^{2}$ Laboratory of Social \\ Cognitive Affective Neuroscience, The \\ University of Hong Kong, Hong Kong; \\ ${ }^{3}$ The State Key Laboratory of Brain \\ and Cognitive Sciences, Hong Kong; \\ ${ }^{4}$ Institute of Clinical Neuropsychology, \\ The University of Hong Kong, Hong \\ Kong; ${ }^{5}$ nstitute for Biomagnetism \\ and Biosignalanalysis, University of \\ Münster, Münster, Germany
}

\begin{abstract}
The observation of pain in others may enhance or reduce self-pain, yet the boundary conditions and factors that determine the direction of such effects are poorly understood. The current study set out to show that visual stimulus awareness plays a crucial role in determining whether vicarious pain primarily activates behavioral defense systems that enhance pain sensitivity and stimulate withdrawal or appetitive systems that attenuate pain sensitivity and stimulate approach. We employed a mixed factorial design with the between-subject factors exposure time (subliminal vs optimal) and vicarious pain (pain vs no pain images), and the within-subject factor session (baseline vs trial) to investigate how visual awareness of vicarious pain images affects subsequent self-pain in the cold-pressor test. Self-pain tolerance, intensity and unpleasantness were evaluated in a sample of 77 healthy participants. Results revealed significant interactions of exposure time and vicarious pain in all three dependent measures. In the presence of visual awareness (optimal condition), vicarious pain compared to no-pain elicited overall enhanced self-pain sensitivity, indexed by reduced pain tolerance and enhanced ratings of pain intensity and unpleasantness. Conversely, in the absence of visual awareness (subliminal condition), vicarious pain evoked decreased self-pain intensity and unpleasantness while pain tolerance remained unaffected. These findings suggest that the activation of defense mechanisms by vicarious pain depends on relatively elaborate cognitive processes, while - strikingly - the appetitive system is activated in highly automatic manner independent from stimulus awareness. Such mechanisms may have evolved to facilitate empathic, protective approach responses toward suffering individuals, ensuring survival of the protective social group.
\end{abstract}

Keywords: observation of pain, approach, defense, pain tolerance

\section{Introduction}

Self-experienced acute pain, the "unpleasant sensory and emotional experience associated with [...] (temporary, real) tissue damage"l on one's own body, evokes enhanced pain sensitivity and withdrawal urges from the pain-inducing stimulus. ${ }^{2}$ Individual pain sensitivity is reflected in measures of pain tolerance, that is, the point at which individuals terminate pain stimulation that they consider unbearable $\mathrm{e}^{3,4}$ and self-reported pain intensity and unpleasantness, respectively, indexing sensory and affective pain evaluations. ${ }^{3}$ Withdrawal responses to pain-inducing stimuli reflect activation of the behavioral defense system, ${ }^{5}$ and may be augmented by the distress elicited by witnessing other individuals in pain (i.e., vicarious pain). ${ }^{4-11}$ Studies reveal that vicarious pain can trigger the behavioral defense system, resulting in decreased self-pain tolerance and increased self-pain intensity and unpleasantness ratings in the observer. ${ }^{3,4-11}$ In
Correspondence: Kati Keuper
Institute for Biomagnetism and Biosignalanalysis, University of Münster, Malmedyweg 15, 48I49 Münster, Germany

Email k.keuper@uni-muenster.de

Tatia M C Lee

Rm 656, The Jockey Club Tower, The University of Hong Kong, Pokfulam Road, Hong Kong

Tel +8523917 8394

Email tmclee@hku.hk 
line with this, exposure to vicarious pain animations has been found to stimulate preparatory motor actions associated with withdrawal movements. ${ }^{7,9}$ Notwithstanding, vicarious pain has also been reported to stimulate the behavioral appetitive system, ${ }^{5,12-19}$ as indexed by decreased self-pain perception ${ }^{4,12}$ and enhanced approach behaviors. ${ }^{19-22}$ For example, Mathur et $\mathrm{al}^{13}$ demonstrated that participants reported higher empathy and willingness to donate money toward individuals depicted to be suffering pain compared to non-suffering individuals. Similarly, Hein et $\mathrm{al}^{12}$ found that witnessing group members receiving pain encouraged individuals to express empathic concern, and bear more self-pain in order to reduce pain stimulation for others. Mechanisms underlying these seemingly contradictory withdrawal versus approach-related effects of vicarious pain on self-pain experience are poorly understood.

The current study investigates how visual awareness during vicarious pain influences self-pain experience as indicated by tolerance, intensity and unpleasantness (Figure 1). A manipulation of visual awareness by presenting stimuli at optimal and subliminal exposure times may yield information on the degree of automaticity required to activate behavioral systems. ${ }^{5,8,23}$ Subliminal visual stimuli (e.g., words, images) are presented so briefly that participants fail to consciously detect their contents above chance. ${ }^{24,25}$ Subliminally evoked behaviors thus operate in the absence of elaborate cognitive processes $^{25,26}$ and reflect rather automatic processes that activate behavioral systems with high priority. Conversely, contents of optimal stimuli can be easily discriminated, and are available to conscious regulation. ${ }^{27}$ There is a large corpus of evidence that withdrawal- and threat-associated, negative material is preferentially distinguished from neutral or positive material at very early processing stages, ${ }^{17,18}$ even in the absence of visual awareness. ${ }^{26,28}$ It is likely that such threat information is automatically extracted from vicarious pain images, $5,8,23,28$ instantly activating the behavioral defense system. ${ }^{5,15}$ Supporting this notion, Yamada and Decety found subliminal negative but not positive words to enhance detection of painful versus neutral facial expressions in visually presented stimuli. Accordingly, it was concluded that defense rather than approach is associated with pain faces. ${ }^{8}$ Similarly, Chiesa et $\mathrm{al}^{23}$ presented participants with subliminal pain or pleasant facial expressions and found that subsequent neutral stimuli were rated as, respectively, less or more likeable, indicating that vicarious pain elicited withdrawal in the absence of visual awareness. To account for both withdrawal- and approach-related responses to vicarious pain, it has been suggested that approach behaviors induced by vicarious pain require more elaborate cognitive processes that suppress initial withdrawal responses. ${ }^{5,15,29}$ However the study by Chiesa et al also reported that pupil dilation in response to subliminally presented facial pain expressions was correlated with empathic concern expressed for the images. As empathetic concern rather reflects approach, overall these findings provide preliminary evidence that subliminal vicarious pain may trigger both defense and appetitive systems in the absence of elaborate cognitive analysis. ${ }^{23}$

Considering findings that optimal vicarious pain modulates self-pain experience ${ }^{10}$ and given that both optimal and subliminal vicarious pain modulate approach and withdrawal reactions, ${ }^{8,23}$ we seek to investigate the role of visual awareness on the relationship between vicarious pain and self-pain experience. Participants were presented with subliminal or optimal vicarious pain or no-pain images before pain tolerance, intensity and unpleasantness to cold-pain were administered. Given previous literature, ${ }^{3,6,9-11}$ it is hypothesized that optimal pain images will elicit decreased self-pain tolerance and increased self-pain intensity and unpleasantness ratings compared to optimal no-pain images. Moreover, it is predicted that there will also be differential effects of pain compared to no-pain stimuli on the subliminal level. ${ }^{8,10,23}$ The direction will yield crucial implications regarding the behavioral systems preferentially activated by vicarious pain images in the absence of visual awareness. Explicitly, if subliminal vicarious pain activates the defense system, it is expected that pain images will also evoke decreased self-pain tolerance and increased self-pain intensity and unpleasantness ratings compared to subliminal no-pain images. In contrast, if the approach system is activated, a reverse pattern is expected whereby pain images evoke increased self-pain tolerance and decreased self-pain intensity and unpleasantness ratings compared to subliminal no-pain images.

\section{Materials and methods Participants and procedures}

Healthy Chinese locals in Hong Kong were recruited through poster advertisement and word-of-mouth. Individuals reporting chronic pain conditions, vasospastic disorders, pain medication intake and physical or mental health disorders were excluded from the study. Participants $(\mathrm{N}=77)$ (Table 1) were randomly distributed across four experimental groups (subliminal pain, subliminal no-pain, optimal pain and optimal no-pain). A one-way ANOVA was conducted that confirmed the four groups to be matched for gender $(\mathrm{F}(3,73)=0.577, p=0.632)$, age $(\mathrm{F}(3,73)=0.224, p=0.879)$ and self-reported pain sensitivity $(\mathrm{F}(3,73)=0.306, p=0.821)$ 
A

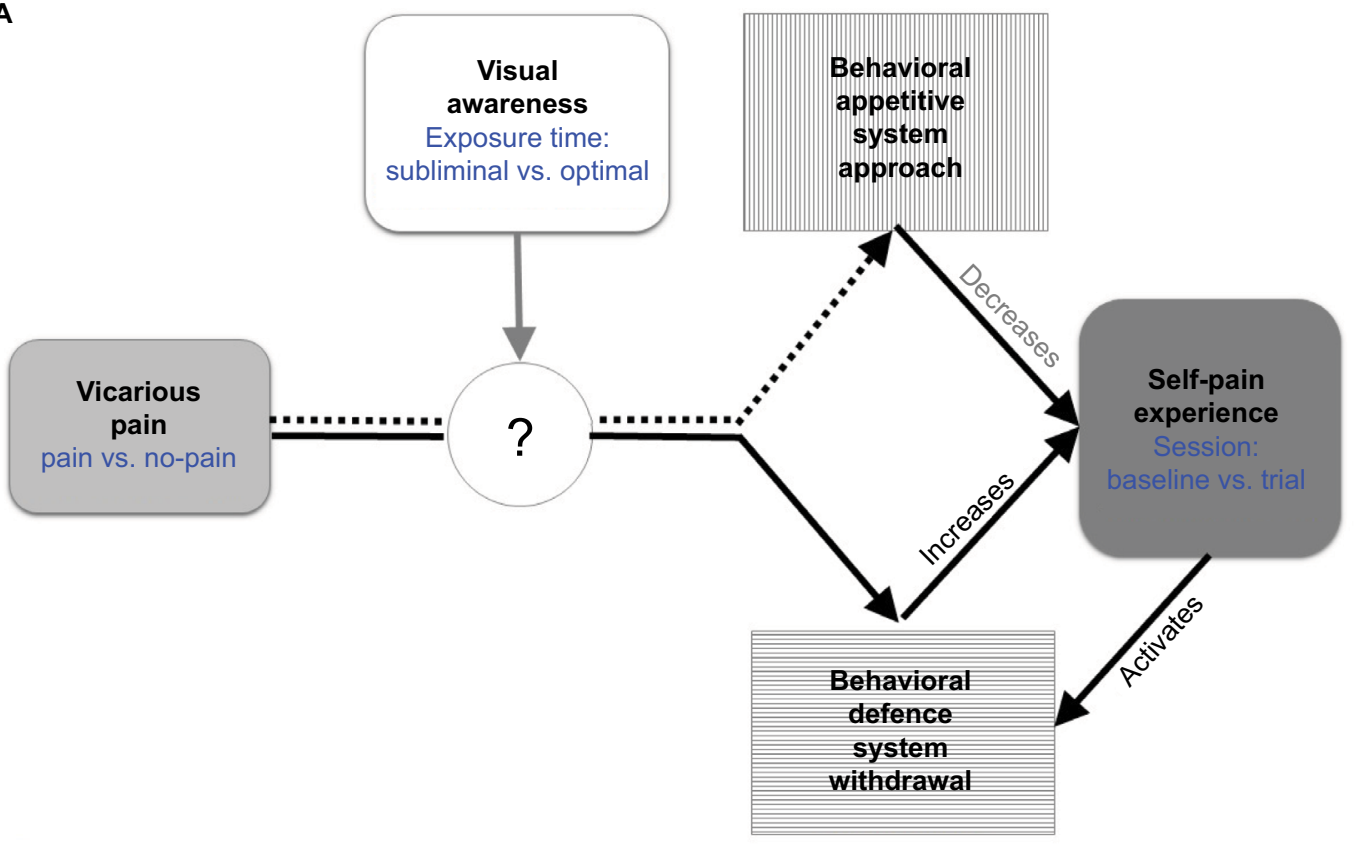

B

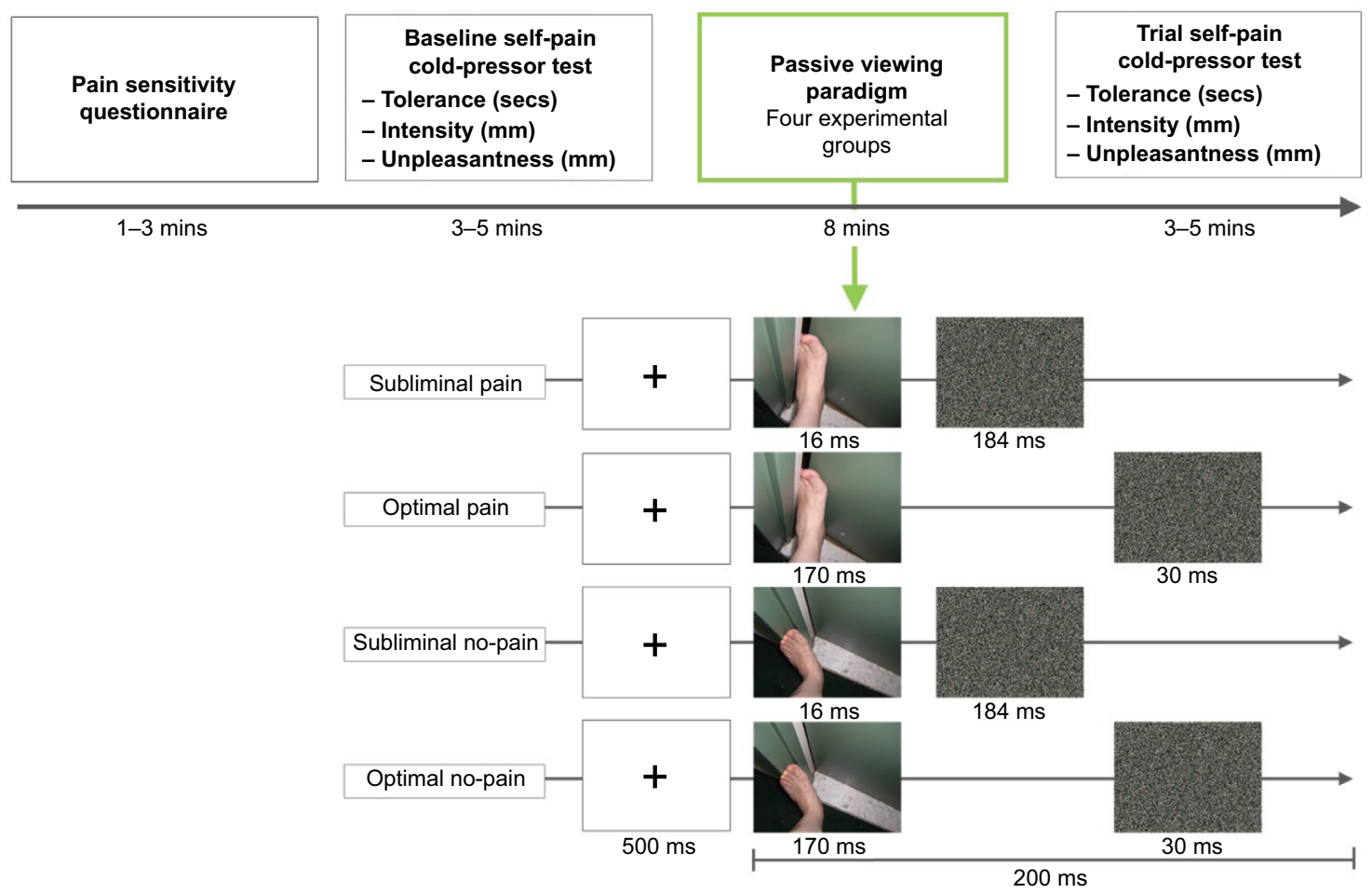

Figure I (A) Relationship among variables of interest. Vicarious pain may activate behavioral defense and behavioral appetitive system, resulting in decreased and increased self-pain experience, respectively. The current study investigates modulatory effects of visual awareness on the relationship between vicarious pain and self-pain experience. Corresponding factors and their factor levels as implemented in the study design are indicated in blue letters. (B) Visualization of Experimental Procedure. After completion of the Pain Sensitivity Questionnaire all participants completed the cold-pressor test (baseline self-pain). Depending on the assigned experimental group (subliminal pain, optimal pain, subliminal no-pain, optimal no-pain) participants were presented one of four passive viewing sequences, in which I 30 images were shown randomly and repeated for five times. To illustrate the trial structure and stimuli included in the passive viewing task, example trials for each experimental group are depicted at the bottom of the figure. Following the passive viewing task, participants again underwent the cold-pressor test (trial self-pain). Pain tolerance, intensity and unpleasantness were obtained as measures for self-pain experience at baseline and trial. 
Table I Demographics and outliers for sample and per condition

\begin{tabular}{llllll}
\hline & Sample & $\begin{array}{l}\text { Sub } \\
\text { Pain }\end{array}$ & $\begin{array}{l}\text { Sub No- } \\
\text { pain }\end{array}$ & $\begin{array}{l}\text { Opt } \\
\text { Pain }\end{array}$ & $\begin{array}{l}\text { Opt } \\
\text { No-pain }\end{array}$ \\
\hline $\begin{array}{l}\text { Total N } \\
\text { Gender N }\end{array}$ & 77 & $18^{*}$ & 20 & 20 & $19^{*}$ \\
$\quad$ & & & & & \\
$\quad$ Males & 42 & $1 \mathrm{I}$ & 9 & 10 & $1 \mathrm{I}$ \\
$\quad$ Females & 35 & $7^{*}$ & $1 \mathrm{I}$ & 10 & $8^{*}$ \\
Age (years), M & 29.39 & 30.06 & 28.60 & 29.95 & 29.37 \\
$\quad($ SD) & $(6.13)$ & $(7.22)$ & $(8.62)$ & $(4.14)$ & $(3.40)$ \\
PSQ, M & 4.70 & 4.43 & 4.73 & 4.78 & 4.75 \\
$\quad(S D)$ & $(1.24)$ & $(1.59)$ & $(0.98)$ & $(0.98)$ & $(0.97)$ \\
\hline
\end{tabular}

Note: *Outliers removed.

Abbreviations: Sub, subliminal; Opt, optimal; PSQ, Pain Sensitivity Questionnaire.

as well as baseline pain tolerance $(\mathrm{F}(3,73)=0.799 p=0.498)$, intensity $(\mathrm{F}(3,73)=1.099, p=0.457)$ and unpleasantness $(\mathrm{F}(3,73)=1.354, p=0.264)$.

\section{Procedure}

Participants were provided with an information sheet and an oral explanation prior to giving their written informed consent to participate in the study. The voluntary nature of their participation was highlighted and they were informed that they could withdraw from the trial at any time without penalty. Please refer to Figure 1B for an overview on the experimental procedure. After giving informed consent, participants were tested individually and first completed demographic information and a pain sensitivity questionnaire (PSQ) ${ }^{30}$ Participants were then exposed to cold-pain using the cold-pressor test (CPT) ${ }^{31}$ whereby baseline self-pain tolerance, intensity and unpleasantness ratings were obtained. According to the experimental group to which they had been randomly allocated, participants were afterward presented one out of four different image sequences in a passive viewing task. They were instructed to carefully attend to all images as they might be asked questions about specific images afterwards. The passive viewing task lasted for $\sim 8$ minutes. Subsequently, participants were again subjected to the CPT. Trial self-pain tolerance, as well as intensity and unpleasantness ratings were obtained. All participants were debriefed upon session completion. Following the guidelines of The Declaration of Helsinki, the study was approved by the Human Research Ethics Committee for Non-Clinical Faculties, The University of Hong Kong.

\section{Design}

This study follows a $2 \times 2 \times 2$ mixed factorial design with the between-subject factors exposure time (optimal vs subliminal presentation) and vicarious pain (pain vs no-pain images) and the within-subject factor session (baseline vs trial). The three dependent variables of self-pain tolerance, intensity and unpleasantness were measured at baseline and trial, that is, before and after exposure to the passive viewing task realizing the experimental groups. Difference scores were calculated for each measure of self-pain by subtracting baseline from trial scores, and then submitted to three separate $2 \times 2$ between-subject ANOVAs with the betweensubject factors exposure time and vicarious pain. Post hoc Bonferroni-corrected independent $t$-tests were conducted for all significant interactions. To investigate robustness of the current data, replications of well-established findings of gender differences in self-pain perception ${ }^{32}$ and correlations between self-reported pain sensitivity and self-pain perception ${ }^{30}$ were undertaken on baseline data. Furthermore, effects of gender, pain sensitivity and age on difference scores of all pain measurements were examined.

\section{Materials}

The PSQ ${ }^{30}$ measures subjective pain susceptibility by asking individuals to rate hypothetical pain scenarios on a scale of 0-10, ranging from no pain to the worst pain imaginable. Scenarios include items such as "imagine you burn your tongue on a very hot drink." The PSQ has a high reliability and validity ${ }^{30}$ and was shown to accurately predict objectiveself-pain experience. ${ }^{30}$ In the current study, the PSQ was administered to avoid systematic between-group differences in self-reported pain sensitivity.

The well-established CPT consists of a bucket filled with $8-10 \mathrm{~cm}$ of ice and water, which is continuously circulated with a pump and held at $\sim 2{ }^{\circ} \mathrm{C} .{ }^{31,33}$ Participants submerged their non-dominant hand into the water, and withdrew when they felt the sensation became intolerable. The time between submersion and retrieval was measured in seconds as pain tolerance. All submersions remained below the safety maximum of 180 seconds. ${ }^{33}$ Upon removal, participants were provided with a towel. Pain intensity and unpleasantness ratings were measured from 0 to $100 \mathrm{~mm}$ on two separate paper visual analog scales (VAS). These ranged from no pain at all to worst pain imaginable and not unpleasant at all to most unpleasant pain imaginable, respectively. VAS have been established in previous pain research to be robust in detecting small changes in self-report pain ratings. ${ }^{34}$

For the passive viewing task, we selected 260 horizontal digital color images $(185 \times 138$ pixels $)$ validated in previous vicarious pain research. ${ }^{35}$ All images depicted human limbs from the first-person perspective, previously blurred with a Gaussian filter to remove gender or age effects. These 
images consisted of 130 matched pain/no-pain image pairs depicting hands and feet in painful and non-painful scenarios (see Figure 1B for an example of the stimulus material) and were identical in luminance and contrast ${ }^{35}$ (luminance $\mathrm{t}(258)=1.149, p=0.251 ;$ red $\mathrm{t}(258)=0.741, p=0.459$; blue $\mathrm{t}(258)=1.251, p=0.212$; green $\mathrm{t}(258)=1.370, p=0.172)$. Scrambled masks were made from each image, rendering its contents unrecognizable with Matlab 2008a. ${ }^{36}$ The script calculated total pixels per image, and randomly changed the position of each pixel while keeping image width and height constant (Figure 1B).

To realize the four experimental groups, four passive viewing sequences were created on E-Prime 2.0 Professional Software, ${ }^{37}$ which differed in exposure time (subliminal or optimal) and vicarious pain (pain or no-pain). For all four sequences, the respective 130 pain or no-pain images were randomly shown after a fixation cross of $500 \mathrm{~ms}$, and repeated once in each of 5 consecutive blocks. Following previous literature, ${ }^{29}$ subliminal images were presented for $16 \mathrm{~ms}$ followed by a mask of $184 \mathrm{~ms}$, while optimal images were presented for $170 \mathrm{~ms}$ followed by a $30 \mathrm{~ms}$ mask (Figure 1B). The images were presented in the center of a 13 inch computer screen with a $1920 \times 1080$ resolution and a viewing distance of $600 \mathrm{~mm}$, corresponding to a visual angle of $4.3 \times 3.3^{\circ}$. These settings are in accordance with those used in previous subliminal research to ensure images remain under the discrimination threshold. ${ }^{29}$ Discrimination tests with all images confirmed that the image contents could not be determined above chance both on single and repeated trials. Eight additional participants indicated by forced-choice response whether subliminal and optimal images were pain or no-pain depictions. ${ }^{25}$ D-prime scores were calculated according to signal detection theory, whereby scores over 1 and close to 0 , respectively, indicate that responses did and did not differ from chance distributions. ${ }^{38,24}$ No significant difference was revealed between responses and chance for subliminal images $(\mathrm{t}(7)=0.541, p=0.605$; d' mean $[\mathrm{SD}]=0.03[0.16])$. In contrast, responses to optimal presentations were above chance $(\mathrm{t}(7)=7.32, p<0.001$, d' mean $[\mathrm{SD}]=2.12[0.81])$. As previous research has shown that repeated subliminal image presentations can improve discrimination, ${ }^{39}$ a further eight participants confirmed that five subliminal repetitions of each image did not elicit above-chance discrimination $(\mathrm{t}(7)=-0.427, p=0.682$, d' mean $[\mathrm{SD}]=-0.01[0.08])$.

\section{Results}

Three $2 \times 2$-way between-subjects ANOVAs with the factors exposure and vicarious pain revealed a significant main effect for both vicarious pain $(\mathrm{F}(1,73)=4.149, p=0.045)$ and exposure $(\mathrm{F}(1,73)=13.506, p<0.001)$ on pain tolerance difference scores (trial - baseline), but not on pain intensity (vicarious pain: $\mathrm{F}(1,73)=0.310, p=0.580$; exposure: $\mathrm{F}(1,73)=0.300, p=0.586$ ) or unpleasantness (vicarious pain: $\mathrm{F}(1,73)=0.967, p=0.329$; exposure: $\mathrm{F}(1,73)=.863, p=0.356$ ) difference scores. Importantly, all three pain measures were subject to significant interactions of vicarious pain and exposure time, indicating that awareness moderates effects of vicarious pain on self-pain perception (pain tolerance: $\mathrm{F}(1,73)=12.378, p=0.001, \eta^{2}=0.145$; pain intensity: $\mathrm{F}(1,73)=8.402, p=0.005, \eta^{2}=0.103$; pain unpleasantness: $\left.\mathrm{F}(1,73)=12.543, p=0.001, \eta^{2}=0.147\right)$. Due to the significant interactions, the observed main effects on pain tolerance scores were not further interpreted. Interactive effects were delineated using Bonferroni-corrected independent $t$-tests.

To test the hypothesis that optimal vicarious pain will activate the behavioral defense system, effects of optimal pain compared to no-pain images were explored. Analyses revealed a significant decrease in pain tolerance (Figure 2A; $\mathrm{t}(37)=4.086, p<0.000)$ and a corresponding increase in pain intensity (Figure 2B; $\mathrm{t}(37)=-1.958, p=0.05$ ) and unpleasant ratings (Figure $2 \mathrm{C} ; \mathrm{t}(37)=-2.091, p=0.043$ ) for optimal pain compared to no-pain images. These findings are in line with an activation of the defense system by optimal vicarious pain.

To investigate the role of awareness during pain observation, the effects of subliminal pain compared to no-pain images were investigated. Furthermore, optimal and subliminal conditions were compared separately for the pain and no-pain condition. No significant difference in pain tolerance was revealed for individuals exposed to subliminal pain compared to no-pain images $(\mathrm{t}(35)=-0.852, p=0.428)$. In the pain condition, participants showed significantly decreased pain tolerance for optimal compared to the subliminal presentations $(\mathrm{t}(36)=4.217, p=0.000)$. Conversely, in the no-pain condition, no significant difference was found for optimal compared to subliminal presentations $(\mathrm{t}(37)=0.147$, $p=0.884$ ). These findings suggest that the effects of vicarious pain on pain tolerance in the sensory pain system require optimal visual awareness.

Contrasting with measures of pain tolerance, significant effects of vicarious pain were found in evaluative sensory and affective pain systems. Compared to optimal exposure, a reverse pattern emerged for the subliminal condition, whereby exposure to subliminal pain compared to no-pain images elicited a significant decrease in pain intensity $(\mathrm{t}(36)=2.143$, $p=0.043$ ) and unpleasantness ratings ( $\mathrm{t}(36)=2.845, p=0.007)$. These patterns resemble activation of the behavioral appeti- 
A

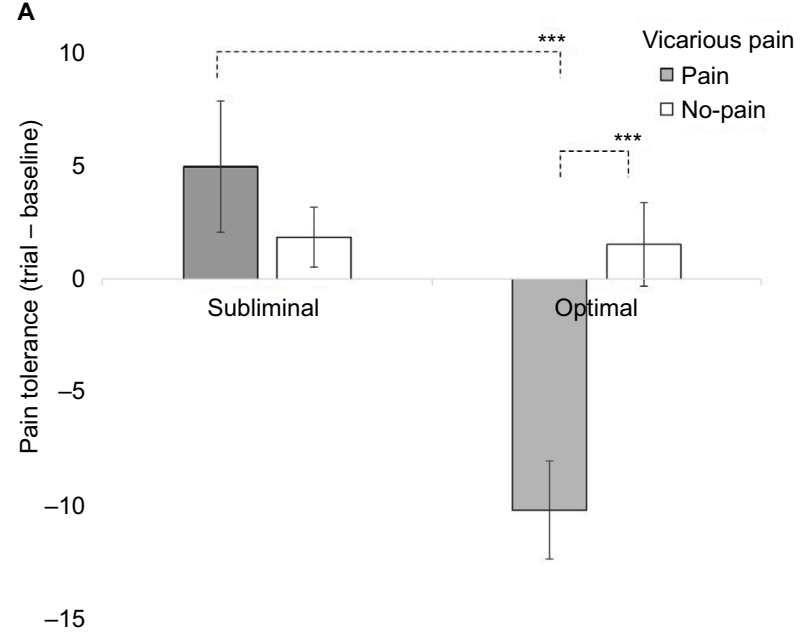

B

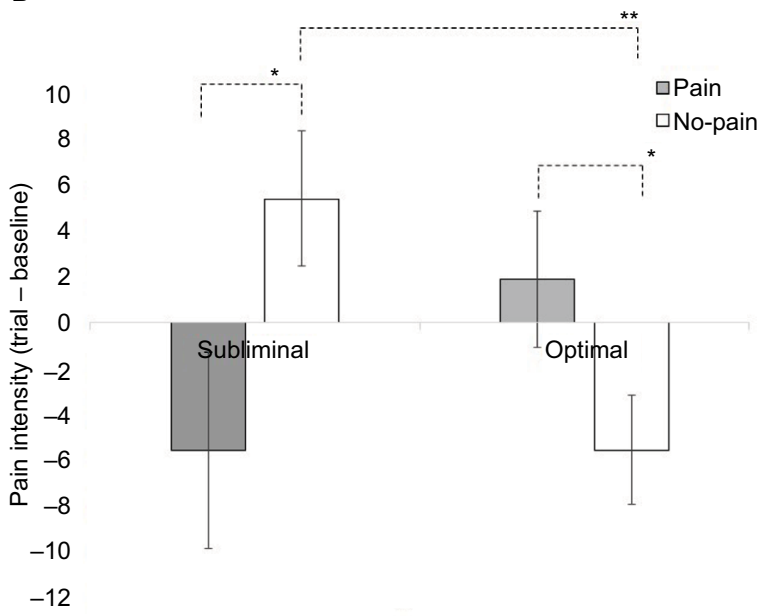

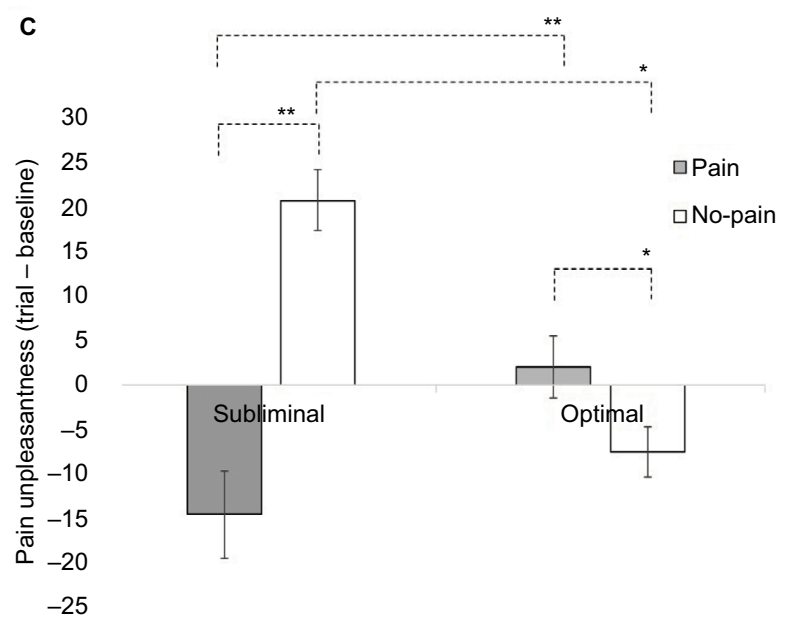

Figure 2 Bar charts depicting mean self-pain difference scores (trial - baseline) per condition for (A) pain tolerance in seconds; (B) pain intensity in millimeters; (C) pain unpleasantness in millimeters. Error bars depict standard errors $(* * * \alpha<0.00$ I, $* * \alpha<0.0$ I, $* \alpha<0.05)$.

tive system during subliminal exposure to vicarious pain. Notably, for pain intensity ratings there was no significant difference for subliminal compared to optimal exposure to pain images $(\mathrm{t}(36)=-1.458, p=0.153)$. In contrast, subliminal compared to optimal no-pain presentations resulted in enhanced intensity ratings $(\mathrm{t}(37)=2.880, p=0.007)$. For pain unpleasantness ratings, there was significant decrease in unpleasantness ratings for subliminal compared to optimal exposure to pain images $(\mathrm{t}(36)=-2.787, p=0.008)$ and a significant increase in unpleasantness ratings for subliminal compared to optimal exposure to no-pain images $(\mathrm{t}(37)=2.165, p=0.037)$. It was concluded that the reverse patterns between subliminal and optimal exposure were driven by the no-pain condition for pain intensity and for both pain and no-pain conditions for pain unpleasantness ratings. These findings reflect distinct visual awareness modulations of pain sensitivity in the evaluative sensory and affective pain system.

\section{Replication of gender and correlational findings}

Independent $t$-tests on baseline data replicated findings of significant differences for genders, with males showing overall decreased sensitivity to cold-pain with increased tolerance and decreased evaluations compared to females (Table 2). ${ }^{32}$

Further replicating prior studies ${ }^{30}$ correlational analyses confirmed that PSQ scores showed significant moderate positive correlations with intensity and unpleasantness ratings, but not tolerance (Table 3). ${ }^{30}$ Similarly, significant positive correlations between intensity and unpleasantness ratings ${ }^{6}$ as well as negative correlations between pain tolerance and both pain evaluations ${ }^{16}$ were replicated.

In contrast to baseline data, difference scores in pain measurements from baseline to trial were not affected by gender (all $p>0.1$ ), pain sensitivity (all $p>0.1$ ) or age (all $p>0.1$ ). Accordingly, within-subject changes in pain perception were 
Table 2 Gender differences in baseline pain measurements: means, SDs, $t$-values and $p$-values

\begin{tabular}{lllll}
\hline & Gender & Mean (SD) & $\begin{array}{l}t \text {-value } \\
(d f)\end{array}$ & $\begin{array}{l}p \text {-value } \\
\text { (one-tailed) }\end{array}$ \\
\hline Tolerance & Female & $29.48(25.85)$ & -2.806 & $0.006^{* *}$ \\
(seconds) & Male & $49.02(35.16)$ & & \\
Intensity $(\mathrm{mm})$ & Female & $58.51(21.49)$ & 1.819 & $0.034^{*}$ \\
& Male & $49.76(20.63)$ & & \\
Unpleasantness & Female & $65.31(20.73)$ & 1.718 & $0.045^{*}$ \\
$(\mathrm{~mm})$ & Male & $56.86(22.14)$ & & \\
\hline
\end{tabular}

Notes: $*^{*} \alpha<0.05,{ }^{* *} \alpha<0.01$.

Abbreviation: $\mathrm{df}$, degrees of freedom

Table 3 Pearson's correlation ( $r$ ) for all baseline pain measurements

\begin{tabular}{llll}
\hline & Tolerance & Intensity & Unpleasantness \\
\hline PSQ scores & 0.067 & $0.318^{*}$ & $0.226^{*}$ \\
Tolerance & $I$ & $-0.34 I^{*}$ & $-0.355^{* * *}$ \\
Intensity & $I$ & $I$ & $0.832^{* * *}$ \\
\hline
\end{tabular}

Notes: $* \alpha<0.05, * * * \alpha<0.001$.

Abbreviation: PSQ, Pain Sensitivity Questionnaire.

not impacted by such factors. Thus, these variables were not included as covariates in the main analyses.

\section{Discussion}

This is the first study to investigate and reveal that visual awareness moderates the impact of vicarious pain on selfpain perception. It was revealed that exposure to optimal pain compared to no-pain images elicited decreased selfpain tolerance and increased intensity and unpleasantness ratings. Reverse patterns were revealed for subliminal pain images, which elicited no changes in self-pain tolerance, and decreased intensity and unpleasantness ratings compared to no-pain images. The discussion offers potential mechanisms for the modulatory role of visual awareness for vicarious pain on self-pain perception and highlights areas of future investigation.

In line with previous research, ${ }^{4,69-11}$ current findings demonstrate that optimal pain compared to no-pain images sensitized both sensory and affective pain systems, eliciting decreased self-pain tolerance ${ }^{4,9,29}$ and increased intensity and unpleasantness ratings. ${ }^{7,10,11}$ Accounting for such findings, the motivational priming hypothesis indicates that vicarious pain enhances pain sensitivity and withdrawal urges through activation of the behavioral defense system. ${ }^{5}, 15$ In line with this hypothesis, neuroscientific data reveal that self-pain and observed pain are encoded by overlapping neural networks, which may act accumulatively during self-pain to enhance the sensory experience. ${ }^{6,729,40}$ In sharp contrast to optimal vicarious pain exposure, the current study found no evidence for activation of the behavioral defense system by subliminal vicarious pain. It is possible that subliminal pain images do not sufficiently activate the behavioral defense system to stimulate the withdrawal responses. In line with these considerations, Godinho et $\mathrm{al}^{10}$ reported that subliminal vicarious pain failed to elicit shared neural patterns with self-pain processing and concluded that subliminal pain images did not influence subsequent self-pain behavior. ${ }^{6,720,29}$ This might explain why, in the current study, exposure to subliminal-pain and no-pain had similar effects on pain tolerance.

However, our data not only argue against an activation of the defense system by subliminal vicarious pain. Strikingly, measures of evaluative sensory and affective pain systems - that is, pain intensity and pain unpleasantness ratings, respectively - revealed reversed effects for subliminal compared to optimal exposure to vicarious pain. Specifically, exposure to subliminal pain compared to no-pain images elicited decreased intensity and unpleasantness ratings. Such pain attenuation corresponds to activation of the appetitive system, ${ }^{5}$ suggesting that in the absence of elaborate conscious processing this system rather than defense may be preferentially activated..$^{6,12,-14,20,28}$ Reduced self-pain evaluations during pain observation may facilitate empathic approach responses that ensure survival of injured members of the social group. ${ }^{12,13}$ Notably, subliminal compared to optimal no-pain images elicited increased self-pain intensity and unpleasantness ratings. However, subliminal compared to optimal pain images elicited decreased unpleasantness ratings but equal intensity ratings. This strongly suggests that visual awareness distinctly impacts sensory and affective pain systems ${ }^{3}$ and is in line with prior studies, which reported no impact of subliminal pain images on self-pain intensity ratings ${ }^{10}$ and instead implicit associations with the affective pain system. ${ }^{23}$

For optimal no-pain, the presence of visual awareness permits individuals to consciously evaluate the safety cues contained in no-pain images as a reference point from which to appraise their self-state. ${ }^{41-43} \mathrm{~A}$ possibly resulting activation of the appetitive system through visual-sensory safety feedback may dominate somatosensory pain feedback, stimulating individuals to downregulate their self-pain evaluations..$^{41,42}$ Vice versa, if safety cues followed by self-pain are not subject to conscious evaluative systems as in the subliminal no-pain conditions, a mismatch of conflicting information (safety vs pain) is created. In the absence of awareness, such conflict- 
ing information may result in pronounced defense responses that cannot be consciously downregulated on the basis of an elaborate evaluation of safety cues. ${ }^{3,19,20}$ Such processes may account for our finding that subliminal compared to optimal no-pain images paradoxically elicit increased selfpain ratings.

The interaction of exposure and vicarious pain on intensity ratings (sensory evaluative system) is explained by differential intensity ratings following optimal vs. subliminal exposure to no-pain, but not to pain images. Conversely, for unpleasantness ratings (affective pain system), effects of exposure time were also found in the pain condition. Attenuated unpleasantness ratings in response to subliminal pain images may reflect intrinsic, preferential activation of the appetitive system, decreasing distress and facilitating approach behaviors toward the suffering individual. ${ }^{4,12,13,19,23}$ In line with this, Chiesa et $\mathrm{al}^{23}$ reported an effect of subliminal facial pain expressions on empathic pupil dilation, suggesting that subliminal vicarious pain evokes empathy. Vicarious pain contains strong cues reflecting that individuals require support and protection. Accordingly, the brain may prioritize the extraction of the affective state of the individual in pain over self-oriented threat information from subliminal images in order to promote survival of the social group. Affective pain cues may thus contribute to stimulating approach behaviors and promoting protective interpersonal relationships. ${ }^{19,21,22,44}$ In this respect, vicarious pain may have a unique status that reflects the inherent social nature of humans. ${ }^{22}$ Taken together, current findings suggest that visual awareness may play a key role in facilitating withdrawal or empathy by modulating the activation of behavioral systems.

The current study is a first step in teasing apart the modulatory effects of visual awareness on associations between vicarious pain and self-pain perception. The replication of well-established gender differences, ${ }^{32}$ pain sensitivity correlations with self-reports for pain ${ }^{30}$ as well as effects of optimal vicarious pain on pain perception ${ }^{4,7,9,29}$ provide a robust framework for the current findings. Direct comparisons between vicarious pain and other negative images are required in order to gain comprehensive understanding of factors modulating activation of behavioral systems. Future investigations may further elucidate neurocognitive mechanisms underpinning the interplay of visual awareness, vicarious pain and selfpain. For example, it would be of interest to see whether individuals who report experiencing pain sensations in their own body upon observing vicarious pain may be more prone to withdrawal or approach responses compared to those who do not perceive such sensations. ${ }^{45}$ Moreover, individuals with empathic deficits or chronic pain frequently exhibit attenuated or augmented self-pain perception, respectively. ${ }^{46,47}$ Thus, implications of the current findings may be further explored in clinical contexts to identify markers and treatment targets of dysfunctional expression of behavioral systems.

In summary, this study confirms that in presence of visual awareness, vicarious pain activates the behavioral defense system, increasing overall self-pain perception. Conversely, in the absence of visual awareness, vicarious pain may activate the appetitive system, decreasing self-pain evaluations and facilitating empathic approach responses. Sensory and affective pain systems may be modulated differentially by visual awareness. These findings suggest that the activation of defense mechanisms by vicarious pain depends on relatively elaborate cognitive processes, while - strikingly - the appetitive system is activated in highly automatic manner, independent from stimulus awareness. Such mechanisms may have evolved to facilitate protective approach responses toward suffering individuals, ensuring survival of the protective social group.

\section{Acknowledgments}

This project was supported by The University of Hong Kong May Endowed Professorship in Neuropsychology. We would like to extend our utmost gratitude to Prof. Jin Fan, Cognitive Neuroscience Laboratory, Queens College, The City University of New York, NY, USA, and Prof. Xiaosi Gu, School of Behavioural and Brain Sciences and Center for Brain Health, The University of Texas at Dallas, TX, USA, for providing our research team with their previously created and validated set of vicarious pain and no-pain images. We deeply appreciate their contribution without which this research paper could not have been written.

\section{Disclosure}

The authors report no conflicts of interest in this work.

\section{References}

1. IASP IAftSoP. Classification of chronic pain. 2 nd ed. Seattle: IASP Press; 1994.

2. Tracey I, Mantyh PW. The cerebral signature for pain perception and its modulation. Neuron. 2007;55(3):377-391.

3. Lamm C, Nusbaum HC, Meltzoff AN, Decety J. What are you feeling? Using functional magnetic resonance imaging to assess the modulation of sensory and affective responses during empathy for pain. PLoS One. 2007;2(12):e1292.

4. Morrison I, Tipper SP, Fenton-Adams WL, Bach P. "Feeling” others' painful actions: the sensorimotor integration of pain and action information. Hum Brain Mapp. 2013;34(8):1982-1998.

5. Gray JA. Three fundamental emotion systems. In: Ekman P, Davidson RJ, editors. The Nature of Emotion: Fundamental Questions. 1994;14:243-247. 
6. Vachon-Presseau E, Martel MO, Roy M, Caron E, Jackson PL, Rainville P. The multilevel organization of vicarious pain responses: effects of pain cues and empathy traits on spinal nociception and acute pain. Pain. 2011;152(7):1525-1531.

7. Vandenbroucke S, Crombez G, Loeys T, Goubert L. Observing another in pain facilitates vicarious experiences and modulates somatosensory experiences. Front Hum Neurosci. 2014;8:631.

8. Yamada M, Decety J. Unconscious affective processing and empathy: an investigation of subliminal priming on the detection of painful facial expressions. Pain. 2009;143(1):71-75.

9. Avenanti A, Paluello IM, Bufalari I, Aglioti SM. Stimulus-driven modulation of motor-evoked potentials during observation of others' pain. Neuroimage. 2006;32(1):316-324.

10. Godinho F, Faillenot I, Perchet C, Frot M, Magnin M, Garcia-Larrea L. How the pain of others enhances our pain: searching the cerebral correlates of 'compassional hyperalgesia'. Eur J Pain. 2012;16(5):748-759.

11. Richter M, Schroeter C, Puensch T, et al. Pain-related and negative semantic priming enhances perceived pain intensity. Pain Res Manag 2014;19(2):69-74.

12. Hein G, Silani G, Preuschoff K, Batson CD, Singer T. Neural responses to ingroup and outgroup members' suffering predict individual differences in costly helping. Neuron. 2010;68(1):149-160.

13. Mathur VA, Harada T, Lipke T, Chiao JY. Neural basis of extraordinary empathy and altruistic motivation. Neuroimage. 2010;51(4):1468-1475.

14. Preston SD, Hofelich AJ. The many faces of empathy: parsing empathic phenomena through a proximate, dynamic-systems view of representing the other in the self. Emot Rev. 2012;4(1):24-33.

15. Goubert L, Vervoort T, Crombez G. Pain demands attention from others: the approach/avoidance paradox. Pain. 2009;143(1-2):5-6.

16. Janssen SA. Negative affect and sensitization to pain. Scand J Psychol. 2002;43(2):131-138

17. Keuper K, Zwitserlood P, Rehbein MA, et al. Early prefrontal brain responses to the hedonic quality of emotional words-a simultaneous EEG and MEG study. PLoS One. 2013;8(8):e70788.

18. Carretié L, Mercado F, Tapia M, Hinojosa JA. Emotion, attention, and the 'negativity bias', studied through event-related potentials. Int J Psychophysiol. 2001;41(1):75-85.

19. Bernhardt BC, Singer T. The neural basis of empathy. Annu Rev Neurosci. 2012;35:1-23.

20. Lamm C, Majdandži J. The role of shared neural activations, mirror neurons, and morality in empathy-a critical comment. Neurosci Res. 2015;90:15-24.

21. Decety J, Bartal IB-A, Uzefovsky F, Knafo-Noam A. Empathy as a driver of prosocial behaviour: highly conserved neurobehavioural mechanisms across species. Phil Trans R Soc B. 2016;371(1686):20150077.

22. Goubert L, Craig KD, Buysse A. Perceiving others in pain: experimental and clinical evidence on the role of empathy. Social Neurosci Empathy. 2011:153-165.

23. Chiesa PA, Liuzza MT, Acciarino A, Aglioti SM. Subliminal perception of others' physical pain and pleasure. Exp Brain Res. 2015;233(8):2373-2382.

24. Williams LM, Liddell BJ, Rathjen J, et al. Mapping the time course of nonconscious and conscious perception of fear: an integration of central and peripheral measures. Hum Brain Mapp. 2004;21(2):64-74.

25. Shevrin H, Panksepp J, Brakel LA, Snodgrass M. Subliminal affect valence words change conscious mood potency but not valence: is this evidence for unconscious valence affect? Brain Sciences. 2012;2(4):504-522.
26. Sweeny TD, Grabowecky M, Suzuki S, Paller KA. Long-lasting effects of subliminal affective priming from facial expressions. Conscious Cogn. 2009;18(4):929-938.

27. Brans K, Koval P, Verduyn P, Lim YL, Kuppens P. The regulation of negative and positive affect in daily life. Emotion. 2013;13(5):926.

28. LeDoux J. The amygdala. Curr Biol. 2007;17(20):R868-R874.

29. Kim EY, Lee SH, Park G, et al. Gender difference in event related potentials to masked emotional stimuli in the oddball task. Psychiatry Investig. 2013;10(2):164-172.

30. Ruscheweyh R, Marziniak M, Stumpenhorst F, Reinholz J, Knecht S. Pain sensitivity can be assessed by self-rating: Development and validation of the Pain Sensitivity Questionnaire. Pain. 2009;146(1): 65-74.

31. Walsh NE, Schoenfeld L, Ramamurthy S, Hoffman J. Normative model for cold pressor test. Am J Phys Med Rehabil. 1989;68(1):6-11.

32. Fillingim RB, King CD, Ribeiro-Dasilva MC, Rahim-Williams B, Riley JL. Sex, gender, and pain: a review of recent clinical and experimental findings. J Pain. 2009;10(5):447-485.

33. Mitchell LA. Cold pressor test. In: Marc G, Rick TJ, editors. Encyclopedia of Behavioral Medicine. Springer-Verlag New York. 2013:455-456.

34. Price DD, McGrath PA, Rafii A, Buckingham B. The validation of visual analogue scales as ratio scale measures for chronic and experimental pain. Pain. 1983;17(1):45-56.

35. Gu X, Liu X, Guise KG, Naidich TP, Hof PR, Fan J. Functional dissociation of the frontoinsular and anterior cingulate cortices in empathy for pain. J Neurosci. 2010;30(10):3739-3744.

36. Mathworks, [homepage on the Internet]. Available from: http://www. mathworks.com/.

37. Psychology Software Tools, Inc., [homepage on the Internet]. Available from: https://www.pstnet.com/.

38. Green D, Swets J. Signal detection theory and psychophysics. 1966. New York. 1966;888:889.

39. Atas A, Vermeiren A, Cleeremans A. Repeating a strongly masked stimulus increases priming and awareness. Conscious Cogn. 2013;22(4):1422-1430.

40. Lamm C, Decety J, Singer T. Meta-analytic evidence for common and distinct neural networks associated with directly experienced pain and empathy for pain. Neuroimage. 2011;54(3):2492-2502.

41. Goubert L, Vlaeyen JW, Crombez G, Craig KD. Learning about pain from others: an observational learning account. J Pain. 2011;12(2): $167-174$.

42. Moseley GL, Gallace A, Spence C. Is mirror therapy all it is cracked up to be? Current evidence and future directions. Pain. 2008;138(1): $7-10$.

43. Longo MR, Betti V, Aglioti SM, Haggard P. Visually induced analgesia: seeing the body reduces pain. J Neurosci. 2009;29(39):12125-12130.

44. Ibáñez A, Hurtado E, Lobos A, et al. Subliminal presentation of other faces (but not own face) primes behavioral and evoked cortical processing of empathy for pain. Brain Res. 2011;1398:72-85.

45. Osborn, J, Derbyshire SWG. Pain sensation evoked by observing injury in others. Pain. 2010;148(2):268-274.

46. Puta, C, Schulz, B, Schoeler, S, et al. Enhanced sensitivity to punctate painful stimuli in female patients with chronic low back pain. $B M C$ Neurol. 2012;12(1):98.

47. Danziger N, Prkachin KM, Willer JC. Is pain the price of empathy? The perception of others' pain in patients with congenital insensitivity to pain. Brain. 2006;129(9):2494-2507. 
The Journal of Pain Research is an international, peer reviewed, open access, online journal that welcomes laboratory and clinical findings in the fields of pain research and the prevention and management of pain. Original research, reviews, symposium reports, hypothesis formation and commentaries are all considered for publication.
The manuscript management system is completely online and includes a very quick and fair peer-review system, which is all easy to use. Visit http://www.dovepress.com/testimonials.php to read real quotes from published authors. 